Original Paper http://ajol.info/index.php/ijbcs http://indexmedicus.afro.who.int

\title{
Existerait-il au sein des peuplements de Lannea microcarpa des individus à caractères botaniques différents dans la zone de Zorgho (Burkina Faso)?
}

\author{
Kadidia SEMDE ${ }^{*}$, Madjelia Cangré Ebou DAO, Boukary Ousmane DIALLO et \\ Souleymane GANABA
}

Département productions forestières/Institut de l'Environnement et de Recherches Agricoles (DPF/INERA), 03 BP 7047 Ouagadougou 03 ; Burkina Faso.

*Auteur correspondant ; E-mail : kadidiasemde@yahoo.fr, Tél : (00226)70740687

\section{RESUME}

La présente étude porte sur la caractérisation botanique de Lannea sp. et Lannea microcarpa et ses Lannea sp. Elle a été conduite à Zorgho, commune située à $110 \mathrm{~km}$ à l'Est de Ouagadougou et a pour objectif de caractériser et de comparer la morphologie des organes des individus. Les échantillons de fleurs et de fruits ont été prélevés entre les mois de mars 2014 et juin 2014 et conservés dans du formol-acide acétique-alcool. Les observations ont concerné la morphologie et la structure florales. Les résultats montrent que les individus de Lannea sp sont à feuilles simples différents de Lannea microcarpa qui sont à feuilles composées. Les analyses révèlent deux types d'inflorescences: les inflorescences femelles à étamines réduites, à pistil développé et celles mâles à étamines développées, à pistil réduit. Il ressort de cette étude la présence Lannea sp au sein des peuplements de Lannea microcarpa. Les investigations pourront se poursuivre sur le plan génétique pour voir si ces individus sont de nouvelles espèces.

(C) 2015 International Formulae Group. All rights reserved.

Mots clés : Caractère, botanique, comparaison, inflorescences, Lannea sp., Burkina Faso

\section{Would there exist within the settlements of Lannea microcarpa individuals with different botanical characters in the zone of Zorgho (Burkina Faso)?}

\section{ABSTRACT}

This study focuses on the botanical characterization of Lannea sp and Lannea microcarpa individuals. The study was conducted at Zorgho city located at $110 \mathrm{~km}$ from the eastern part of Ouagadougou. The objective was to characterize and compare the morphology of the organs. Samples of flowers and fruits were collected between March 2014 and June 2014 and stored in alcohol-acetic acid and formalin. Observations were made on the floral morphology and structure. Results showed that individuals of Lannea sp have simple leaves, different from those of Lannea microcarpa which were compound leaves. Analysis revealed two types of inflorescences: the female inflorescences with reduced stamens and developed pistils and the male inflorescences with developed stamen and reduced pistils. This study brought out the presence of Lannea sp. 
individuals within the populating of Lannea microcarpa. Other investigations at the genetic level could be carried on to see whether these individuals are new species.

(C) 2015 International Formulae Group. All rights reserved.

Keywords: Botany, character, comparison, inflorescences, Lannea sp., Burkina Faso.

\section{INTRODUCTION}

Lannea est un genre appartenant à la famille des Anacardiaceae et regroupe plus de 40 espèces (Ouattara et al., 2011). Cinq espèces sont rencontrées au Burkina Faso: Lannea microcarpa, Lannea acida, Lannea vélutina, Lannea edulis, Lannea barteri, Lannea kerstingii. Elles se caractérisent par des feuilles composées, imparipennées, avec 4 à 9 paires de folioles (Arbonnier, 2009). Parmi elles, Lannea microcarpa (raisinier) aux fruits verdâtres qui sont des drupes et devenant rougeâtres pourpres, est la plus utilisée au Burkina Faso. Elle joue de multiples rôles dans nos sociétés (Belem et al., 2008; Thiombiano et al., 2010 ; Dro et al., 2013; Sérémé, 2014). En effet, sur le plan alimentaire, les fruits sont consommés crus lorsqu'ils sont mûrs ou transformés en jus (Yaméogo et al., 2013). Malheureusement, elles subissent de pressions dues à de multiples facteurs (Yélémou et al., 2009; Baggnian et al, 2014).

Cependant, dans la commune de Zorgho, en zone nord soudanienne, des populations locales ont réussi à donner des noms à des écotypes de Lannea microcarpa à cause de leurs caractères morphologiques. Ils sont issus du même milieu écologique que Lannea microcarpa. Dans les localités où ils sont présents, les populations les décrivent comme des arbres différents des autres espèces de Lannea et trouvent que les fruits ont la même qualité nutritionnelle que ceux de Lannea microcarpa. Ils ont tout de même un nom local «Kankanm-wombsiba»(en mooré) en raison de leurs caractéristiques physiques et leur importance mystique, avec des stades phénologiques qui semble coïncider à ceux de Lannea microcarpa. Les inventaires forestiers nationaux réalisés en 1980, 1983 par le ministère de l'environnement et du développement durable n'ont pas fait cas de ces individus. Les différences entre ces individus (nommés morphotyes de Lannea microcarpa ou Lannea sp.) et Lannea microcarpa vont-elles au-delà en touchant les pièces florales qui abritent les organes reproducteurs en charge de la pérennisation de l'espèce? Compte tenu des implications sociales, il est essentiel d'entreprendre des études pour contribuer à leur caractérisation précise. L'objectif de cette étude est de caractériser sur le plan botanique la morphologie des individus de Lannea sp. et les comparer à ceux de Lannea microcarpa pour comprendre les différences et les ressemblances afin de montrer leur existence et de contribuer à la conservation et à la gestion des peuplements de Lannea.

\section{MATERIEL ET METHODES Site d'étude}

L'étude a été conduite au Burkina Faso dans la commune de Zorgho $\left(12^{\circ} 15 \mathrm{~N} ; 0^{\circ} 36\right.$ $\mathrm{W} ; 310 \mathrm{~m}$ d'altitude) située à $110 \mathrm{~km}$ à l'Est de Ouagadougou. D'une superficie de 453,481 $\mathrm{km}^{2}$, elle a un climat de type nord soudanien et se caractérise par une végétation à savane arbustive évoluant sur des sols ferrugineux tropicaux lessivés, localisés dans le Nord et le Nord-Est et les vertisols au Sud et au SudOuest. La saison des pluies s'étale de 4-5 mois (juin à octobre) tandis que la saison sèche dure 7-8 mois (novembre à mai). Les précipitations annuelles varient entre 500 et $900 \mathrm{~mm}$ et sont très régulièrement caractérisées par une mauvaise répartition spatio-temporelle. Les mois les plus pluvieux sont généralement ceux de juillet et août. La Figure 1 indique les différents sites d'études dans la commune de Zorgho.

\section{Méthodes}

Dans la présente étude, les méthodes suivantes ont été employées: il s'agit des 
observations directes, à la loupe et au microscope. Sur le terrain, les observations ont portées sur le tronc, la forme et le type de feuilles des arbres de Lannea sp. de NaabMayooghin (NLs), de Mamousyorgo1(MLs) et de Mamousyorgo2 (MaLs) et de Lannea microcarpa pris comme témoin. Les observations à la loupe ont concerné la morphologie des inflorescences, des boutons floraux et la taille des organes reproducteurs pour identifier le caractère sexuel des individus, et la forme des fruits. Les échantillons d'inflorescences et d'infrutescences ont été récoltés pendant la période de reproduction de mars 2014 à juin 2014. Trente inflorescences ont été prélevées au bas, au milieu et au sommet du houppier des individus pour des observations à la loupe $(\mathrm{GX} 2,5)$ et pour des mensurations. Pour déterminer la structure florale, cinquante boutons floraux par inflorescence ont été disséqués pour le comptage du nombre de sépales, de pétales, d'étamines et de pistil. L'absence ou la présence des grains de pollen dans les anthères des étamines a été réalisée grâce à des observations au microscope électronique (GX40) ; l'utilisation du rouge de Carmin a permis de connaître la viabilité des pollen. Ainsi, deux lots d'anthères par individu ont été constitués. Le premier lot a été écrasé, monté avec de l'eau entre lame et lamelle et observé au microscope. Le deuxième a été trempé dans la solution du rouge de Carmin (le rôle est de colorer les grains de pollen viables en rouge vif et les grains de pollen non viables ou cellules mortes en rose) pendant $24 \mathrm{~h}$, écrasé, monté entre lame et lamelle et observé au microscope. Toutes les observations sont suivies de photographies pour illustrer les organes.

\section{Analyse statistique}

Les données collectées pour les différents paramètres ont été traitées avec le logiciel statistique XLSTAT 2013. Les données sont soumises à une analyse de variance (ANOVA). Les moyennes des variables ont été comparées en utilisant le test de Newman Keuls au seuil de probabilité de $5 \%$.

\section{RESULTATS}

Caractérisation du port végétal des individus de Lannea sp. et de Lannea microcarpa

La Figure 2 montre le port des individus. Les individus présentent les caractéristiques suivantes: NLs est un arbre dont la hauteur mesure $11 \mathrm{~m}$ et un diamètre à hauteur de poitrine (dhp) de $55 \mathrm{~cm}$. Il est situé à proximité d'un canal d'écoulement d'eau. Le tronc est court, grise blanchâtre avec des écailles en plaquettes (Figure 2a). MLs est lui aussi arborescent avec une hauteur de $10 \mathrm{~m}$ et un dhp de $37 \mathrm{~cm}$. Il est relativement jeune avec une écorce grise, assez lisse et un tronc lisse (Figure 2b). MaLs est arbustif de $7 \mathrm{~m}$ de hauteur avec un dhp de $25 \mathrm{~cm}$, l'écorce est écailleuse, grise et un tronc non droit. Il est porté par un tronc principal de Lannea microcarpa dont la ramification commence à partir du tronc principal à une cinquantaine de centimètre du sol. Ces deux individus imbriqués portent de nettes différences qui sont observables aussi bien sur le plan physiologique que morphologique. Lannea microcarpa (Figure 2d) porte un tronc droit, écailleux, avec une hauteur de $14 \mathrm{~m}$, un dhp de $80 \mathrm{~cm}$ et une écorce grise à blanchâtre.

\section{Morphologie foliaire}

La Figure 3 indique la morphologie foliaire des individus. Les observations directes montrent les caractéristiques foliaires suivantes : les feuilles de NLs sont simples, de forme ovale ou obovale à sommet et à base arrondis (Figure 3a). Les nervures secondaires sont transversales subopposées avec 4 à 5 paires de nervures par feuille. MLs est caractérisé par des feuilles simples, larges, arrondies et veloutées. La feuille montre un limbe à bord entier avec des nervures secondaires variant de 4 à 6 paires par feuille ou parfois plus (Figure 3b). Quant à MaLs, il se caractérise par des feuilles simples qui sont plus ou moins arrondies avec 4 à 5 paires de nervures secondaires par feuille (Figure 3c). 
Lannea microcarpa porte des feuilles composées, opposées avec 3 à 6 paires de folioles ovales, une terminale (Figure $3 \mathrm{~d}$ ).

\section{Caractéristiques des inflorescences}

La Figure 4 illustre les caractéristiques des inflorescences chez les individus. Ainsi, NLs porte des inflorescences en grappe rameuse constituées de boutons floraux allongés, à pédoncules longs disposés de façon lâche sur le rameau (Figure 4a). Chez MLs, les inflorescences sont en grappe rameuse composées de boutons floraux plus ou moins arrondis à pédoncules courts, disposés en grappe serrée (Figure 4b). Celles de MaLs sont en grappe rameuse avec des boutons floraux allongés à pédoncules longs disposés de façon lâche sur le rameau (Figure 4c). Chez Lannea microcarpa les inflorescences sont groupées sur des ramaux constituées de boutons floraux arrondis à pédoncules courts disposés en grappe serrée (Figure 4d).

Le test de variance effectué sur les valeurs obtenues pour le paramètre taille des inflorescences montre une différence significative entre les différentes sections du houppier de Lannea microcarpa et MaLs d'une part et d'autre part entre Lannea microcarpa et NLs (Tableau 1).

\section{Morphologie des organes reproducteurs}

La Figure 5 représente la morphologie et la structure des organes des différents individus. Les mesures $(\mathrm{mm})$ effectuées sur les organes reproducteurs donnent les résultats suivants: Le pistil (organe reproducteur femelle) de NLs de $1,55 \mathrm{~mm}$ de taille moyenne est formé d'un ovaire volumineux contenant un ovule surmonté de 4 longs stigmates aux extrémités sombres avec des styles allongés (Figure 5a). Le pistil de MLs de taille réduite $(0,89 \mathrm{~mm})$ est formé d'un ovaire contenant un ovule surmonté de 3 courts stigmates (Figure 5b). Chez MaLs, la taille moyenne du pistil est de $1,59 \mathrm{~mm}$ et est formé d'un ovaire volumineux surmonté de 4 longs stigmates aux extrémités sombres formés de longs styles, contenant un ovule volumineux (Figure 5c). La fleur de Lannea microcarpa montre un pistil de taille moyenne $(0,88 \mathrm{~mm})$, formé d'un ovaire avec un ovule réduit (Figure 5d).

Concernant les étamines (organe reproducteur mâle), elles sont réduites chez NLs avec une taille moyenne de $0,76 \mathrm{~mm}$ (Figure 5a'). Chez MLs, les fleurs portent des étamines volumineuses $(1,7 \mathrm{~mm})$ qui sont caractérisées par 4 fentes longitudinales (Figure 5b'). Les étamines de MaLs sont formées d'anthères à petites fentes (Figure $\left.5 c^{\prime}\right)$ avec une taille moyenne de 0,78 mm; celles de Lannea microcarpa sont bien formées $(1,7 \mathrm{~mm})$ avec des fentes à déhiscence longitudinale (Figure 5d').

Aussi, les mensurations des différents organes reproducteurs ont permis de faire sortir l'histogramme représenté par la Figure 6 qui montre des différences significatives $(\mathrm{p}<0,05)$ entre la taille des organes de Lannea microcarpa et celle de NLs et MaLs.

\section{Caractéristiques des fruits}

Cependant, les observations montrent que tous les fruits sont de forme ovale, portent des excroissances ( 2 à 3 ) à leur sommet appelées «cornes» ou «dents ». Ces fruits sont de couleur verte lorsqu'ils ne sont pas mûrs et deviennent pourpres à maturité

\section{Étude des pièces florales}

La coupe anatomique des fleurs des individus de Lannea sp. et de Lannea microcarpa effectuée sous la loupe montre des pièces florales disposées de façon alterne les unes après les autres : NLs est constitué d'un calice dialysépale composé de 4 sépales libres, une corolle dialypétale à 4 pétales libres, un androcée formé de 8 étamines et un gynécée. Celle de MLs est formée d'un calice dialysépale composé de 4 sépales libres, d'une corolle dialypétales à 4 pétales libres, un androcée formé de 8 étamines longues, 8 carpelles soudés, une anthère à déhiscence longitudinale. L'individu MaLs montre un calice dialysépale composé de 4 sépales libres, une corolle dialypétale formée de 4 pétales libres, d'un androcée formé de 8 étamines 
courtes, 8 carpelles soudés et un gynécée. Conformément aux autres individus, Lannea microcarpa est caractérisé par un calice dialysépale composé de 4 sépales libres, d'une corolle dialypétale formée de 4 pétales libres, d'un androcée, 8 étamines longues, 8 carpelles soudés, une anthère à déhiscence longitudinale, un pistil réduit formé d'un ovaire surmonté de 3 styles et stigmates courts. L'ensemble des individus montre de l'extérieur vers l'intérieur les pièces florales suivantes: les sépales, les pétales, les étamines, les carpelles et l'ovaire. La formule florale se présente alors comme suit : $4 \mathrm{~S}+$
$4 \mathrm{P}+10 \mathrm{E}+\underline{8 \mathrm{C}} . \mathrm{S}=$ sépales, $\mathrm{P}=$ pétales, $\mathrm{E}=$ étamines, $\mathrm{C}=$ carpelles. $\mathrm{De}$ cette formule se dégage le diagramme florale (Figure 7).

\section{Morphologie des grains de pollen :}

La Figure 8 illustre la morphologie des grains de pollen. L'observation au microscope ordinaire des étamines des fleurs des individus montre des fragments de membrane d'anthère chez NLs. Ces mêmes observations ont été faites chez MaLs. Les anthères de MLs et Lannea microcarpa montrent des grains de pollen de forme ovale. Avec le rouge de Carmin, ces pollens ont viré au rouge vif.

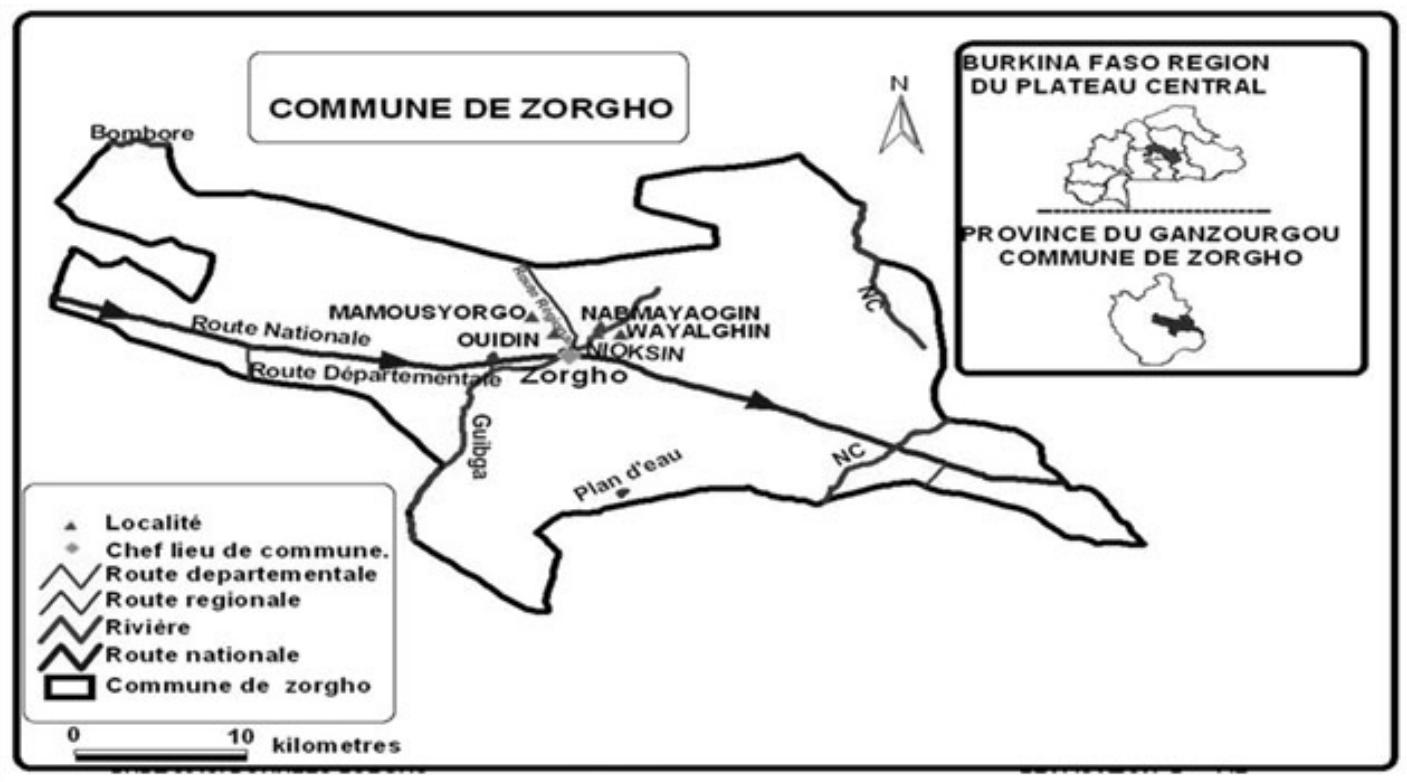

Figure 1: Localisation des sites d'étude dans la commune urbaine de Zorgho.

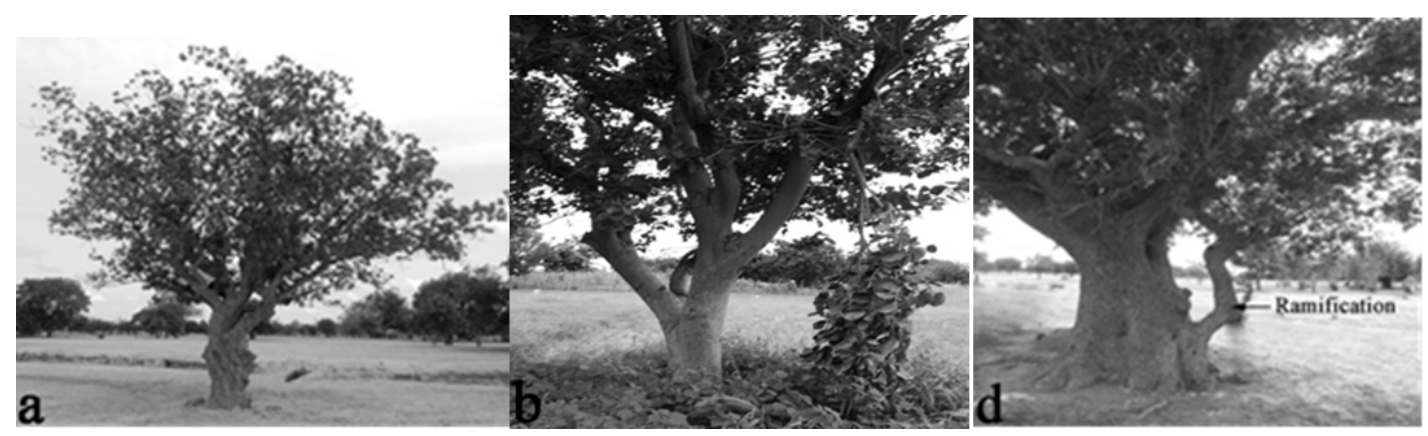

a : Lannea sp de Naab-Mayooghin, b : Lannea sp de Mamousyorgo1

ramification = Lannea $\mathrm{sp}$ de Mamousyorgo2, $\mathrm{d}:$ pied de Lannea microcarpa

Figure 2: Port végétal des individus de Lannea sp et de Lannea microcarpa. 


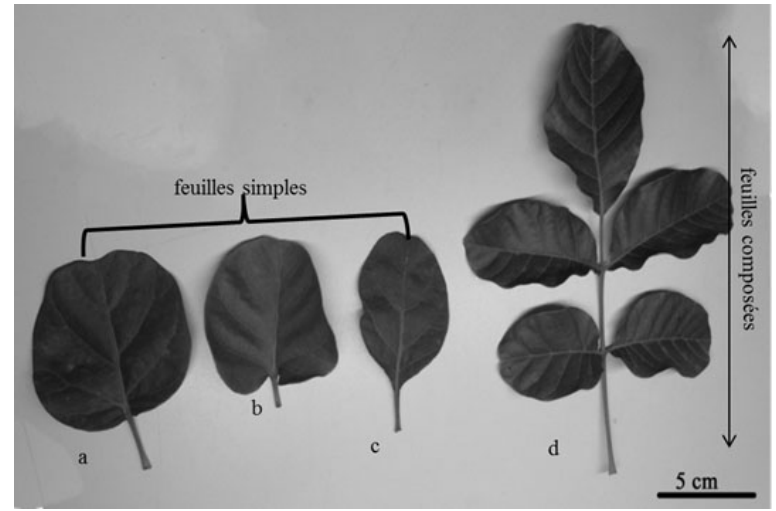

Feuille de Lannea sp de Naab-Mayooghin, b : Feuille de Lannea sp de Mamousyorgo1 $\mathrm{c}:$ Feuille de Lannea sp de Mamousyorgo2, d : Feuille de Lannea microcarpa

Figure 3: Morphologie foliaire des individus de Lannea sp et de Lannea microcarpa.

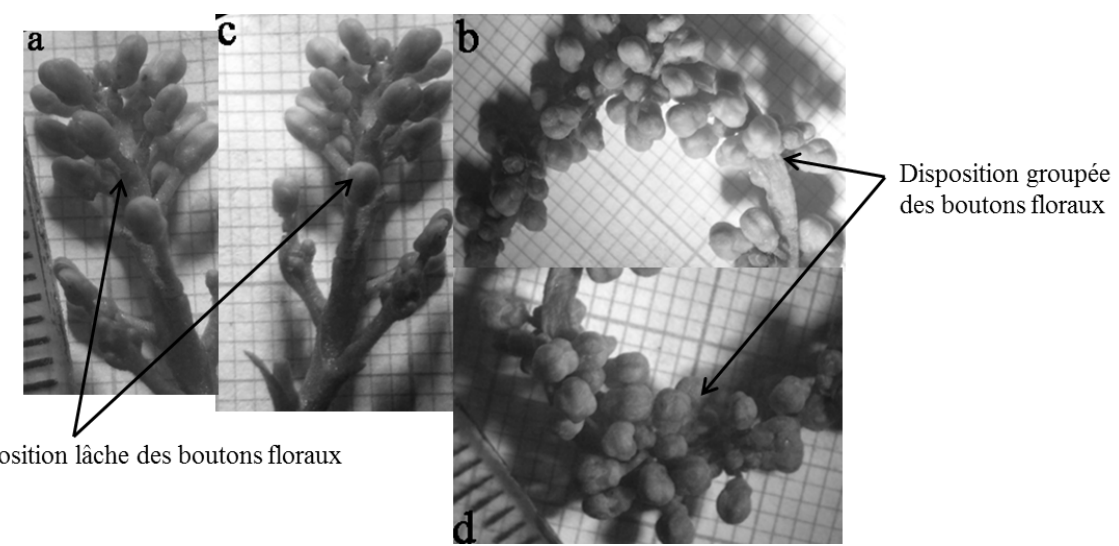

a: inflorescence du pied de Naab-Mayooghin ; b : inflorescence du pied de Mamousyorgo 1

$\mathrm{c}$ : inflorescence du pied de Mamousyorgo2, $\mathrm{d}$ : inflorescence du pied de Lannea microcarpa

Figure 4: Disposition des boutons floraux sur les rameaux des différents individus.

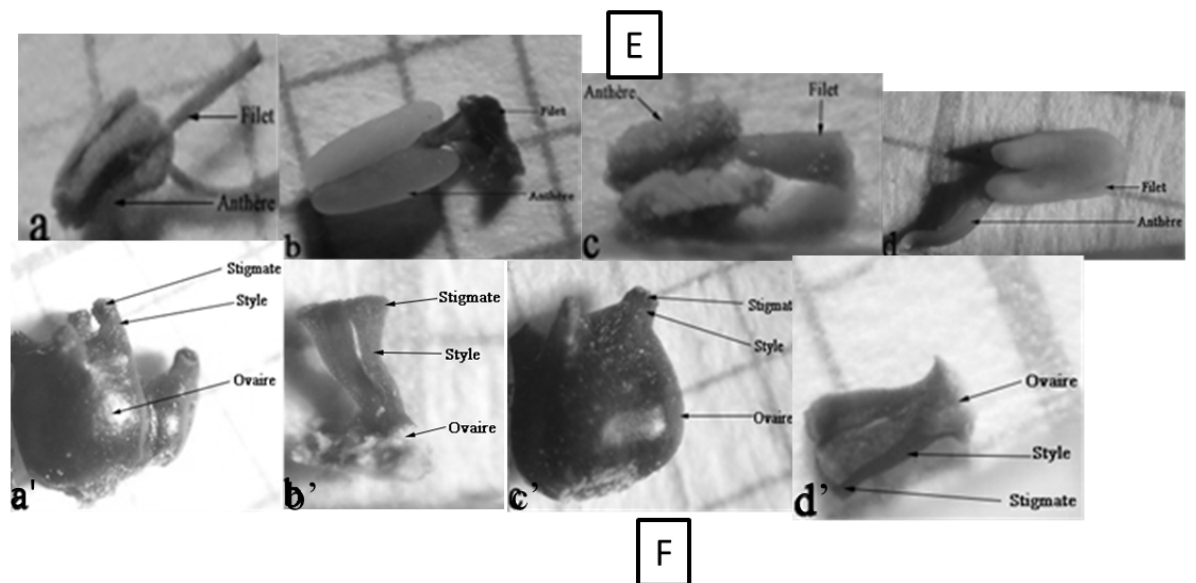

a : pistil de la fleur de Naab-Mayooghin ; b : pistil de la fleur de Mamousyorgo1

c : pistil de la fleur de Mamousyorgo2; d : pistil de la fleur de Lannea microcarpa ;

a' : étamine de la fleur de Naab-Mayooghin ; b': étamine de la fleur de Mamousyorgo1

c': étamine de la fleur de Mamousyorgo2; d': étamine de la fleur de Lannea microcarpa ;

Figure 5: Morphologie et structure des organes reproducteurs femelles (E) et mâles (F). 


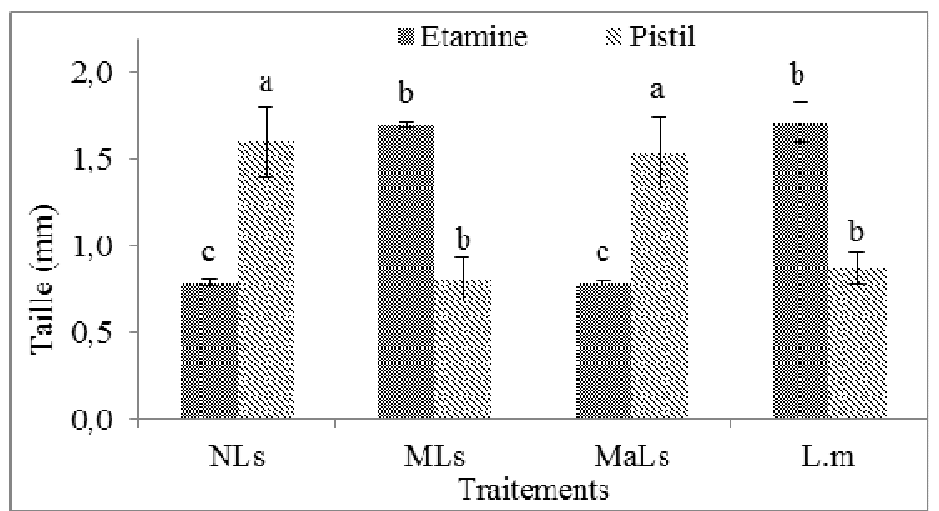

$\mathrm{N}=$ Naab-Mayooghin, M=Mamousyorgo1, Mamousyorgo2, Ls=Lannea $\mathrm{sp}, \mathrm{L} . \mathrm{m}=$ Lannea microcarpa

Figure 6: Comparaison de la taille des organes reproducteurs (étamine et pistil : $\mathrm{p}<0,0001$ ) en fonction des individus des différents sites.

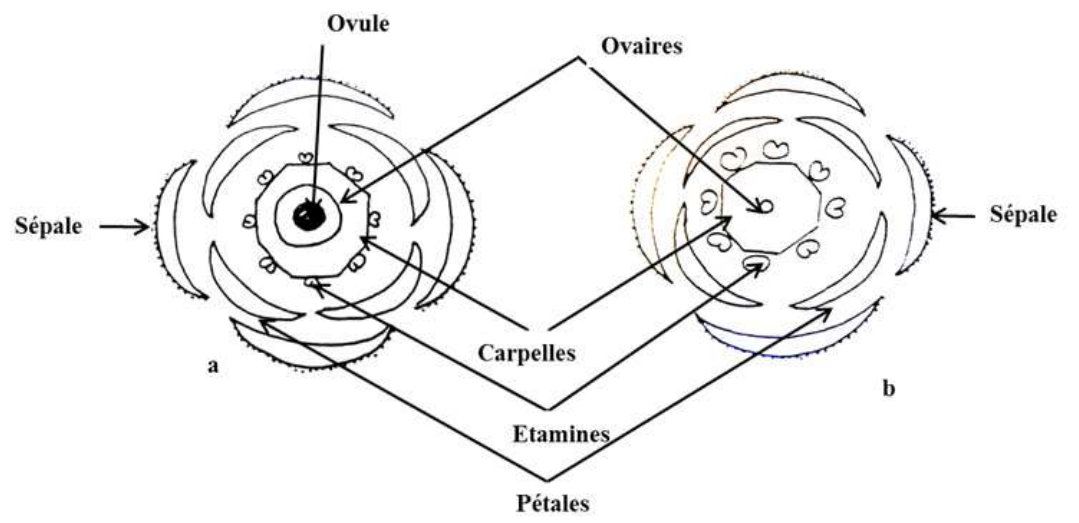

$\mathrm{a}=$ NLs et MaLs $; \mathrm{b}=$ MLs et Lannea microcarpa

Figure 7: Diagrammes floraux des Lannea sp et Lannea microcarpa.

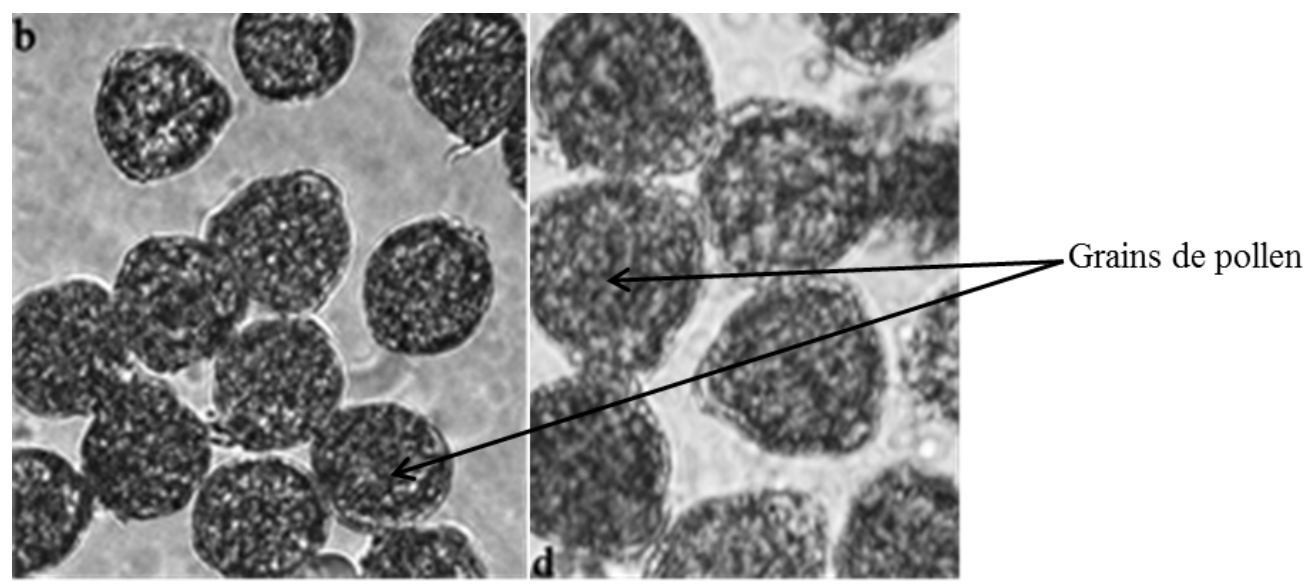

Figure 8: Grains de pollen de MLs ( $\mathrm{b}=$ MLs : Lannea sp de Mamousyorgo1), et de Lannea microcarpa $(\mathrm{d})$. 
Tableau 1: Comparaison de la taille des inflorescences de l'ensemble des individus en fonction de leur section

\begin{tabular}{lccc}
\hline Traitements & Bas & Milieu & Haut \\
\hline L.m & $3,12 \pm 0,7^{\mathrm{a}}$ & $6,20 \pm 1,13^{\mathrm{a}}$ & $7,71 \pm 1,00^{\mathrm{a}}$ \\
NLs & $2,29 \pm 0,00^{\mathrm{b}}$ & $3,04 \pm 0,00^{\mathrm{b}}$ & $2,56 \pm 0,00^{\mathrm{b}}$ \\
MLs & $4,12 \pm 0,69^{\mathrm{a}}$ & $6,37 \pm 1,13^{\mathrm{a}}$ & $7,56 \pm 0,98^{\mathrm{a}}$ \\
MaLs & $1,69 \pm 0,70^{\mathrm{b}}$ & $2,19 \pm 1,13^{\mathrm{b}}$ & $2,06 \pm 01,01^{\mathrm{b}}$ \\
Niveau de signification & $\mathrm{P}=0,004$ & $\mathrm{P}<0,0001$ & $\mathrm{P}<0,0001$ \\
\hline
\end{tabular}

L.m = Lannea microcarpa, $\mathrm{N}=$ Naab-Mayooghin, Mamousyorgo 1, Mamousyorgo2, Ls= Lannea $\mathrm{sp}$

Pour une même colonne les valeurs affectées des lettres différentes sont statistiquement différentes au seuil de $5 \%$ selon le test de Newman-Keuls (SNK) $(\mathrm{p}<0,05)$.

\section{DISCUSSION Port végétal}

Les plantes étudiées montrent des caractéristiques du port végétal différentes les unes des autres. En effet, NLs porte un tronc écailleux qui est observé chez MaLs et Lannea microcarpa. Par contre, le tronc de MLs est lisse, différent de Lannea microcarpa. Cette différence s'explique par l'écart d'âge qui pourrait exister entre ces arbres. Ces résultats corroborent avec ceux de Arbonnier (2009) qui ont montré que la nature $\mathrm{du}$ tronc d'un arbre varie en fonction de sa durée d'existence, plus l'arbre est jeune, plus il présente un tronc lisse. MLs pourrait être plus jeune que les autres individus. Des différences ont été également observées au niveau de la morphologie et du type foliaires.

\section{Morphologie foliaire}

Les résultats ont montré que NLs, MLs et MaLs se caractérisent par des feuilles simples, larges, arrondies au sommet alors que les feuilles de Lannea microcarpa sont composées constituées de folioles ovales et pointues au sommet. En effet, les travaux de Sacandé (2007), Arbonnier (2009) ont montré que les espèces de Lannea microcarpa sont constituées de feuilles composées. Les caractéristiques des feuilles des individus de Lannea sp. sont différentes de celles de Lannea microcarpa. En effet, la différence foliaire qui existe entre les espèces de Lannea microcarpa et les individus de Lannea sp. pourrait s'expliquer par des raisons génétiques. Puisque les différences se situent au niveau des feuilles, on pourrait penser à des mutations chromosomiques qui ont fait passer ces individus de $2 \mathrm{n}$ chromosomes à $\mathrm{Xn}$ chromosomes avec X supérieur ou égal à 3. Il s'agit d'une première étude sur les individus de Lannea sp. dont les résultats pourront servir de bases de données à la recherche des connaissances scientifiques desdits individus.

Caractéristiques florales (inflorescences, boutons floraux et organes reproducteurs)

La taille des inflorescences varie non seulement pour les sections du houppier d'un même individu mais aussi d'un individu à l'autre. A cet effet, deux types d'inflorescences sont observés. Le premier caractérisé par des inflorescences courtes (NLs et MaLs) est constitué de boutons floraux allongés disposés en hélice lâche sur le rameau, un pistil développé et des étamines réduites. Par contre le deuxième type (chez MLs) porte des inflorescences longues caractérisé par des boutons floraux arrondis, disposés en hélice serrée sur les rameaux, avec des étamines plus développées et un ovaire réduit. Les caractéristiques florales de ce dernier ressemblent à celles de Lannea microcarpa qui sont différentes de celles de NLs et de MaLs. Ces différentes caractéristiques montrent l'existence d'un polymorphisme au sein des individus.

En effet, la taille réduite du pistil et celle développée des étamines chez MLs et Lannea microcarpa s'expliquerait par le fait que ces individus sont des pieds mâles. Par contre la forme développée du pistil et celle réduite des étamines des fleurs de NLs et MaLs montrent que ces pieds sont femelles. On peut dire que la présence des pieds mâles et femelles témoigne l'existence des deux caractères sexuels au sein des individus de Lannea sp. Ces résultats confirment ceux de 
Diallo et al. (2006) qui ont pu déterminer le caractère sexuel de certains Anacardiaceae grâce à ces techniques d'études.

\section{Caractéristiques des fruits}

Les observations ont montré que les fruits des individus de Lannea sp. sont des drupes comestibles de forme ovale et ne diffèrent pas de ceux de Lannea microcarpa. En effet, ces résultats confirment ceux de Kouyaté (2005) et Arbonnier (2009) qui ont montré que les fruits de Lannea microcarpa sont des drupes qui occupe une place alimentaire importante au sein de la société burkinabé. Des résultats analogues ont été trouvés par Yunus et al. (2013), Agbogan et al. (2015) dans beaucoup de pays de l'Afrique de l'Ouest.

\section{Caractéristiques des grains de pollen}

Les pollens sont des organes qui entrent dans la détermination des caractères sexuels chez les plantes. Ainsi, dans les anthères des étamines de MLs, des pollens bien développés virant au rouge vif ont été observés. Ces mêmes résultats ont été observés chez Lannea microcarpa. La couleur rouge vif montre que ces pollens sont viables. Ceci confirme les résultats de Diallo et al. (2006) sur les travaux des fleurs de Sclerocarya birrea. En effet, l'existence des pollens viables dans les anthères des étamines et la forme réduite du pistil des fleurs de MLs et Lannea microcarpa montrent que ces pieds porteraient des fleurs hermaphrodites. Ces résultats corroborent avec ceux de Yélémou (2008) sur les caractéristiques des fleurs de Piliostigma reticulatum. Par contre, chez NLs et MaLs des fragments d'anthères ont été observés dans les anthères des étamines, ce qui montre l'absence de pollens et confirmerait le caractère sexuel femelle chez ces individus. Dao (2012) ont également identifié le caractère sexuel chez certaines espèces grâce à la caractérisation des grains de pollen.

D'où l'existence d'un dimorphisme sexuel au sein des individus de Lannea sp. Cependant, on note que les fleurs hermaphrodites et les fleurs femelles de ces individus sont portées par des pieds différents ; on pourrait les qualifier de plantes gynodioïques.

\section{Conclusion}

L'étude a permis de caractériser et de montrer la présence des individus de Lannea sp. au sein des peuplements de Lannea microcarpa avec une morphologie foliaire qui se diffère. Deux types de caractères sexuels ont été identifiés chez les individus de Lannea sp. Aucune différence n'a été observée entre les fruits desdits individus $\mathrm{sp}$ et ceux de Lannea microcarpa. Les données obtenues sont certes d'une importance capitale car elles constituent une avancée notable dans l'obtention des données scientifiques sur ces individus, mais elles sont insuffisantes pour conclure sur leur originalité. Cependant, l'étude ouvre des axes de perspectives de recherche sur ces ressources forestières, ce qui pourrait contribuer à enrichir la diversité génétique du pays et peut être un atout dans leur régénération et leur gestion.

\section{CONFLIT D'INTÉRÊT}

Les auteurs déclarent qu'ils n'ont aucun conflit d'intérêt.

\section{CONTRIBUTIONS DES AUTEURS}

KS s'est occupé de la collecte des données, de leurs analyses, de leurs traitements et de la rédaction du document; MCED s'est occupé de la biologie de la reproduction des plantes; OBD s'est occupé de la génétique; SG a contribué à l'encadrement en écologie végétale.

\section{REMERCIEMENTS}

Les auteurs remercient la population de la commune de Zorgho pour leur collaboration dans la réalisation du travail, Dalla Mamadou pour la réalisation de la carte et l'ensemble des lecteurs anonymes pour leurs suggestions et recommandations dans l'amélioration de la qualité de l'article.

\section{REFERENCES}

Agbogan A, Tozo K, Wala K, Bellefontaine R, Dourma M, Akpavi S, Woegan YA, Dimobe K, Akpagana K. 2015. Structure des populations de Sclerocarya birrea, Lannea microcarpa et Haematostaphis barteri au nord du Togo. Journal of Animal and Plant Sciences, 25(2): 38713886 
Arbonnier M. 2009. Arbres, arbustes et lianes des zones sèches d'Afrique de l'Ouest; livre CIRAD, MNHN, UICN, p. 541.

Baggnian I, Adam T, Adamou MM, Chaibou I, Mahamane A. 2013. Structure et dynamique de la végétation ligneuse juvénile issue de la régénération naturelle assistée (RNA) dans le Centre-Sud du Niger. Int. J. Biol. Chem. Sci., 8(2): 649665. DOI http://dx.doi.org/10.4314/ijbcs.v8i2.22

Belem B, Olsen CS, Theilade I, Bellefontaine R, Guinko S, Lykke AM, Diallo A, Boussim JI. 2008. Identification des arbres hors forêt préférés des populations du Sanmatenga (Burkina Faso). Bois et Forêts des Tropiques 298 (4): 1-12

Dao CEM. 2012. Biologie et écologie de la reproduction sexuée d'une Caesalpinioideae (Leguminoseae): Piliostigma reticulatum (D.C.) Hochst. Université Polytechnique de BoboDioulasso (UPB) Institut du Développement Rural (IDR), Burkina Faso ; Thèse de doctorat, p.127.

Diallo BO, Bastide B, Poissonnet M, Dao MCE, Sanou J, et Key MH. 2006. Mise en évidence d'une androdioecie et d'une hétérostigmatie chez Sclerocarya birrea (A.Rich.) Hochst. Fruit, 61(4): 1-8. DOI: 10.1051/fruits:2006023

Dro B, Soro D, Koné MW, Bakayoko A, Kamanzi K. 2013. Evaluation de l'abondance de plantes médicinales utilisées en médecine traditionnelle dans le Nord de la Côte d'Ivoire Journal of Animal and Plant Sciences, 17(3): 26312646.

Kouyaté MA. 2005. Aspects ethnobotaniques et étude de la variabilité morphologique, biochimique et phenologique de detarium microcarpum guill et perr (Mali). Thèse de Doctorat, Universyteit Gent; p. 207.

Ouattara L, Koudou J, Zongo C, Barro N, Sawadogo A, Bassole IHN, Ouattara AS, and Traore AS. 2011. Antioxidant and antibacterial activites of three species of Lannea from Burkina Faso. Journal of Applied Sciences 11 (1): 157-162 DOI: $10.3923 /$ jas.

Sacandé M. 2007. Lannea microcarpa Engl. Seed Leaflet; article Forest et Landscape Denmark Hørsholm Kongevej 123: 112970.

Séréme A, Millogo RJ, Guinko S, Nacro M. 2014. Micropropagation of a West African wild grape (Lannea microcarpa). Int. J. Biol. Chem. Sci., 8(3): 862-870 ; DOI: http://dx.doi.org/10.4314/ijbcs.v8i3.3

Thiombiano DNE, Lamien N, Dibong SD, et Boussim I. 2010. Etat des peuplements des espèces ligneuses de soudure des communes rurales de Pobé-Mengao et de Nobéré (Burkina Faso) Journal of Animal \& Plant Sciences, 9(1): 1104- 1116.

Yaméogo G, Yelemou B, Boussim IJ, et Traore D. 2013. Gestion du parc agroforestier du terroir de Vipalogo (Burkina Faso) : contribution des ligneux à la satisfaction des besoins des populations. Int. J. Biol. Chem. Sci., 7(3): 1087-1105.

DOI: http://dx.doi.org/10.4314/ijbcs.v7i3.16

Yelemou B, Yameogo G, Bationo BA, Millogo-Rasolodimby J, Hien V. 2008. Biologie florale et mode de reproduction sexuée de Piliostigma reticulatum (D.C.) Hochst. Int. J. Biol. Chem. Sci., 2(3): 281291.

Yunus MM, Zuru AA, Faruq UZ and Aliero AA. 2013. Assement of Physicochemical Properties of Biodiesel from African Grapes (Lannea microcarpa Engl. \& K.Krause). Nigerian Journal of Basic and Applied Science 21(2):127-130. DOI: http://dx.doi.org/10.4314/njbas.v21i2.7

Zerbo P., Millogo J R., Nacoulma-Ouedraogo O G., Vandamme P. 2007. Contribution à la connaissance des plantes médicinales utilisées dans les soins infantiles en pays San, au Burkina Faso. Int. J. Biol. Chem. Sci., 1(3): 262-274. 